\title{
Evaluation of color vision and contrast sensitivity in diabetic patients without retinopathy
}

\begin{abstract}
Purpose: The purpose of this study is to evaluate the color vision and contrast sensitivity in diabetic patients without diabetic retinopathy, and to assess the color vision and contrast sensitivity in every diabetic patient before the occurrence of diabetic retinopathy and make it an integral part of routine examination.
\end{abstract}

\begin{abstract}
Materials and methods: A hospital based descriptive cross-sectional study was conducted at Aziz-Fatimah Hospital Faisalabad including 50 patients of age between 30 to 55 years. Information regarding duration of diabetes, diabetes status (controlled/uncontrolled), types of the diabetes was recorded. After taking complete history the visual acuity was measured with tumbling E chart and subjective refraction was performed. Then dilation fundus examination was performed with $+78 \mathrm{D}$ lens and fundus photograph was taken to evaluate diabetic retinopathy. On the next day Color vision and contrast sensitivity of the patients were assessed with Ishihara test and Pelli-Robson Chart Respectively.
\end{abstract}

Results: There were $44 \%$ males and $56 \%$ females with the mean age of $48.42 \pm 5.15$ years Normal contrast sensitivity was observed in $34 \%(n=17)$ of the patients. However, $30 \%$ $(n=15)$ of the patients had reduced contrast sensitivity, $28 \%(n=14)$ of the patients had poor contrast sensitivity and only $8 \%(n=4)$ patients had severe visual impairment. Color vision was observed normal in $68 \%(n=34)$ patients while $24 \%(n=12)$ of the patients had impaired color vision and only $8 \%(n=4)$ of the patients had sever visual impairment. There were $42 \%(n=21)$ diabetic patients with $>5$ years history of diabetes, $38 \%(n=19)$ of diabetic patients with 5-10 years history of diabetes and $20 \%(n=10)$ of diabetic patients had $<10$ years history of diabetes. Type 1 diabetes were observed in $46 \%(n=23)$ and type 2 diabetes were observed in $48 \%(n=24)$. There were only $6 \%(n=3)$ of diabetic patients with dietary control. Most of the patients had controlled diabetes $64 \%(n=32)$ while uncontrolled diabetes were observed in $36 \%(n=18)$ patients.

Conclusion: There were highly significant association of Status of Diabetes (Controlled/ uncontrolled) and duration of diabetes with color vision and contrast sensitivity. There was no significant association of age and gender with color vision and contrast sensitivity. This study was helpful in detecting the early visual function compromised due to diabetes before the occurrence of diabetic retinopathy.

Keywords: Color vision, contrast sensitivity, diabetic retinopathy, diabetes mellitus, visual function
Volume 9 Issue $3-2019$

\author{
Rabia Saeed,' Iqra Iqbal, ${ }^{2}$ Javaria Asif Bajwa, ${ }^{3}$ \\ Sadia Falak ${ }^{4}$ \\ 'Optometrist, Aziz Fatima Hospital, Faisalabad, Pakistan \\ ${ }^{2}$ Optometrist, University of Faisalabad, Madina Teaching Hospital, \\ Pakistan \\ ${ }^{3}$ Optometrist, King Edward Medical University, Mayo Hospital \\ Lahore, Pakistan \\ ${ }^{4}$ Assistant Professor, School of Pharmacy, University of \\ Faisalabad, Pakistan
}

Correspondence: Rabia Saeed, Optometrist, Aziz Fatima Hospital, Faisalabad, Pakistan, Email saeedrabia082@gmail.com

Received: May 14, 2019 | Published: May 17, 2019

\section{Introduction}

Diabetes mellitus is a common disease with devastating effects characterized by increased high glucose level due to defect in insulin production, insulin action or both. Diabetes mellitus occurs when the pancreas cannot produce enough insulin or the cell in the body has resistance to insulin. ${ }^{1}$ The most common type of diabetes is type 1 $(5 \%)$ and type 2 diabetes is $(95 \%)$. The estimated number of people over 18 years of age with diagnosed and undiagnosed diabetes is 30.2 million in the United States. ${ }^{2}$ Among the most populated countries of the world Pakistan ranks eighth in the prevalence of diabetes. In Pakistan about 6.2 million populations are suffering from diabetes and one in every third diabetic patients has diabetic eye disease. ${ }^{3}$ The diabetes mellitus disturbs the physiology of the retinal cells and its pathogenesis. Retinal neuro-degeneration such as alteration in the retinal ganglion cells and inner retinal neurons can cause various forms of visual defects such as decreased contrast sensitivity and impaired color vision and temporal perception. All these changes occur before changes in vascular morphology and visual acuity. ${ }^{4}$ Approximately $7-29 \%$ of diabetic patients attending general medical outpatient department have diabetic retinopathy and two-thirds of diabetic have an increased risk of visual impairment. ${ }^{5}$ According to the World Health organization there are 285 million people are visually impaired and contrast sensitivity is one of the most common leading cause of visual impairment. Worldwide, Diabetic retinopathy with impaired vision, decrease color vision and contrast sensitivity is the leading cause of legal blindness, Between 20 to 74 year of age but it can be prevented through proper glycemic control. ${ }^{4}$

Many studies have established that individuals with diabetic retinopathy can present with impairment of color vision and the severity of impaired color vision increases with increase in the severity of diabetes and the duration of diabetes. ${ }^{2}$ The description and appreciation of colors depend upon the receptors ability on retina i.e. rods and cones. Rods are responsible for day and night vision while cones are responsible for color vision. ${ }^{6}$ The color vision impairment due to any ocular disease is known as acquired color vision deficiency. This acquired form of impaired color vision results in the inability to distinguish similar shades of a color. In the whole world about $8 \%$ of the male and $0.5 \%$ of female are affected with acquired color vision deficiency. ${ }^{4}$ Diabetic mellitus patients may also experienced gradual 
decrease in Contrast sensitivity which may adversely impact on the daily activities of life such as reading, driving, watching, recognizing faces etc. Decrease in contrast sensitivity depends on the severity or stages of diabetic retinopathy.

This study emphasizes to measure the color vision and contrast sensitivity of every diabetic patient because it is a useful diagnostic tool for early detection of abnormalities in retinal functions. It may also help in analyzing the relationship between metabolic control and retinal function. In our region, many studies have been done on color vision and contrast sensitivity of diabetic patients which were mostly related to diabetic retinopathy, but my study investigates the early color vision and contrast sensitivity changes in diabetic patients at a risk of developing clinically detectable diabetic retinopathy.

\section{Material and methods}

This cross-sectional study was conducted at Out-patient clinic of Ophthalmology department of Aziz Fatimah Hospital Faisalabad from December 2018 to March 2019. All the diabetic patients without diabetic retinopathy irrespective of age and gender were included in this study. Patients with non- diabetic status, visual acuity less than $6 / 12$, any congenital diseases, cataract, glaucoma, ocular surface changes, any eye surgery and who were unable to understand or answer interview questions were excluded from the study. A written informed consent was taken from every patient before collecting data. Patient identity and their data were kept confidential. Only researcher had access to their data. No economic burden was put on patients. Participants had full right to withdraw at any time during the study. Data were collected from the patients with the help of self designed Performa. The Performa was designed to collect information about demographic, exposure and outcome variables of patients such as duration of diabetes, type of diabetes, diabetic status (controlled/ uncontrolled), Snellen visual acuity without retinopathy, color vision and contrast sensitivity of patients were noted. A brief ocular history was also taken about the number of visit in eye department, history of glasses or any eye surgery. After the history taken visual acuity of both eyes was measured with Snellen visual acuity chart at six meter distance. After the visual acuity assessment and best possible refractive correction, dilate the patients for fundus examination. After the full dilation, fundus examination was done with the help of $+78 \mathrm{D}$ lens. After that, fundus photographs were taken to rule out the diabetic retinopathy. If the patients had no diabetic retinopathy the status of color vision and contrast sensitivity were assessd on the very next day. Color vision in each eye was assessed by using ishihara 24 plates test in which reading from 1- 15 plates determine the normality or defectiveness of color vision. If the patients read 13 or more than 13 plates the color vision is recorded as normal. If the 9 or less than 9 plates were read normally, the color vision is considered as deficient. If 6 or less than 6 plates read normally the color vision is considered as severely impaired. Contrast sensitivity was measured by using Pelli-Robson Contrast Sensitivity Chart. Normal score of contrast sensitivity was 2 , i.e. $100 \%$. The contrast sensitivity was measured at a distance of 1 meter (40 inches) with the patients wearing their best corrected distance correction. Normal score of contrast sensitivity was 2 , i.e. $100 \%$. Score less than 2 were considered reduced contrast sensitivity. Score less than 1.5 were abnormal and considered a poor contrast sensitivity and a score less than 1 indicate a visual disability or sever visual impairment.

SPSS version 20 was used for statistical analysis. Descriptive statistics was explored by using frequency and percentages for qualitative variables and means, standard deviation for quantitative variables. The correlation of diabetic status (controlled/uncontrolled), duration of diabetes and types of diabetes with color vision and contrast sensitivity were explored by using Chi- square test. PValue $<0.05$ was taken as significant.

\section{Results}

A total of 50 patients were included in the study. Mean age of the patients was $48.42 \pm 5.15$ years. The frequency of females was slightly higher $56 \%(\mathrm{n}=28)$ as compared to males $44 \%(\mathrm{n}=22)$. Normal contrast sensitivity was observed in $34 \%(\mathrm{n}=17)$ of the patients. However, $30 \%$ $(n=15)$ of the patients had reduced contrast sensitivity, $28 \%(n=14)$ of the patients had poor contrast sensitivity and only $8 \%(n=4)$ patients had severe visual impairment. Color vision was observed normal in $68 \%(n=34)$ patients while $24 \%(n=12)$ of the patients had impaired color vision and only $8 \%(n=4)$ of the patients had sever visual impairment. There were $42 \%(n=21)$ diabetic patients with $>5$ years history of diabetes, $38 \%(\mathrm{n}=19)$ of diabetic patients with $5-10$ years history of diabetes and $20 \%(\mathrm{n}=10)$ of diabetic patients had $<10$ years history of diabetes. Type 1 diabetes were observed in $46 \%(\mathrm{n}=23)$ and type 2 diabetes were observed in $48 \%(n=24)$. There were only $6 \%$ $(n=3)$ of diabetic patients with dietary control. Most of the patients had controlled diabetes $64 \%(n=32)$ while uncontrolled diabetes were observed in $36 \%(\mathrm{n}=18)$ patients (Table 1$)$.

By using chi-square test, insignificant results of diabetes controlled were observed with age $\left(\mathrm{P}_{\text {chi }}-\right.$ value 0.215$)$ and with gender $\left(\mathrm{P}_{\text {chi }}-\right.$ value $0.254)$. However, insignificant association of age with color vision $\left(\mathrm{P}_{\text {chi }}-\right.$ value 0.510$)$ and contrast sensitivity $\left(\mathrm{P}_{\text {chi }}-\right.$ value 0.314$)$ and gender with color vision $\left(\mathrm{P}_{\text {chi }}\right.$-value 0.441$)$ and contrast sensitivity $\left(\mathrm{P}_{\text {chi }}-\right.$ value 0.111$)$ were also observed. Chi-square test showed a highly significant association of color vision $\left(\mathrm{P}_{\text {chi }}-\right.$ value 0.00$)$ and contrast sensitivity $\left(\mathrm{P}_{\text {chi }}-\right.$ value 0.00$)$ with the duration of diabetes. The patient having diabetes more than 10 years had more chance of color vision impairment and reduced contrast sensitivity (Figure 1) (Figure 2). The Chi-square test also showed a highly significant association of color vision $\left(\mathrm{P}_{\text {chi }}-\right.$ value 0.00$)$ and contrast sensitivity $\left(\mathrm{P}_{\text {chi }}-\right.$ value 0.00 ) with the status of diabetes (controlled/uncontrolled). Whereas, Color vision impairment is significantly associated with type of diabetes $\left(\mathrm{P}_{\text {chi }}-\right.$ value 0.002$)$ but contrast sensitivity was not showed as much significant association with the type of diabetes $\left(\mathrm{P}_{\text {chi }}-\right.$ value 0.050 ). Type 2 diabetes had less chance of color vision impairment as compared to type 1 diabetes (Table 2).

Table I Baseline characteristics of the patients $(n=50)$.

\begin{tabular}{lll}
\hline & & $\mathbf{N}(\%)$ \\
\hline Age & Mean age & $48.42+5.15$ \\
Gender & Male & $22(44 \%)$ \\
& Female & $28(56 \%)$ \\
Duration of Diabetes & \\
& Less than 5 year & $21(42 \%)$ \\
5 To I0 Year & $19(38 \%)$ \\
II to 15 year & $10(20 \%)$ \\
Type of Diabetes & \\
Type I & $23(46 \%)$ \\
Type 2 & $24(48 \%)$ \\
\hline
\end{tabular}




\begin{tabular}{ll} 
Table Continued & N(\%) \\
\hline Dietary control & $3(6 \%)$ \\
Diabetic Control & \\
Controlled & $32(64 \%)$ \\
Un Controlled & $18(36 \%)$ \\
ColorVision & \\
Normal & $34(68 \%)$ \\
Impaired & $12(24 \%)$ \\
Sever Impairment & $4(8 \%)$ \\
Contrast Sensitivity & $17(34 \%)$ \\
Normal & $15(30 \%)$ \\
Reduced Contrast & $14(28 \%)$ \\
Poor Contrast & $4(8 \%)$ \\
Sever visual Impairment &
\end{tabular}

\section{Discussion}

Contrast sensitivity measurement is the good screening tool for the detection of early visual changes in diabetic patients without diabetic retinopathy. Contrast sensitivity test is the good indicator of retinal health before the occurrence of diabetic retinopathy or the progression of the disease. Contrast sensitivity may also be impaired in the presence of normal visual acuity. My study finding showed a highly significant association (P-value 0.00 ) of contrast sensitivity with the duration of diabetes. My findings concurrent with previous study conducted by Vaibhavee and Manisha showed that a significant association ( $\mathrm{P}$ value $0.05)$ of contrast sensitivity functions with the duration of diabetes ${ }^{5}$. My study also found a highly significant association (P- value 0.00 ) of contrast sensitivity with the uncontrolled diabetes. The result of my study also showed the similar results with other study, that there was a significant association ( $\mathrm{P}$ value less than 0.05 ) of status of diabetes (Controlled/Uncontrolled) with contrsast sensitivity 5 . Hence, it was concluded that contrast sensitivity function was compromised as the blood glucose level fluctuates.

Table 2 Status of contrast senstivity with general variables of patients $(n=50)$

\begin{tabular}{|c|c|c|c|c|c|}
\hline & \multicolumn{5}{|c|}{ Contrast Sensitivity } \\
\hline & Normal & Reduced Contrast & Poor Contrast & Sever visual Impairment & P-Value \\
\hline Variables & $\mathrm{n}(\%)$ & $n(\%)$ & $n(\%)$ & $n(\%)$ & \\
\hline \multicolumn{6}{|l|}{ Age } \\
\hline Mean $48.42+5.15$ & & & & & 0.314 \\
\hline \multicolumn{6}{|l|}{ Gender } \\
\hline Male & II (22\%) & $6(12 \%$ & $3(6 \%)$ & $2(4 \%)$ & \\
\hline Female & $6(12 \%)$ & $9(18 \%)$ & II (22\%) & $2(4 \%)$ & 0.111 \\
\hline \multicolumn{6}{|l|}{ Duration } \\
\hline Less than 5 year & $14(28 \%)$ & $4(8 \%)$ & $3(6 \%)$ & $0(0 \%)$ & \\
\hline $5-10$ year & $3(6 \%)$ & $9(18 \%)$ & $7(14 \%)$ & $0(0 \%)$ & 0 \\
\hline II-I5 year & $0(0 \%)$ & $2(4 \%)$ & $4(8 \%)$ & $4(8 \%)$ & \\
\hline \multicolumn{6}{|l|}{ Type of Diabetes } \\
\hline Type I & $3(6 \%)$ & $8(16 \%)$ & $9(18 \%)$ & $3(6 \%)$ & \\
\hline Type 2 & II(22\%) & $7(14 \%)$ & $5(10 \%)$ & $\mathrm{I}(2 \%)$ & 0.5 \\
\hline Dietary Control & $3(6 \%)$ & $0(0 \%)$ & $0(0 \%)$ & $0(0 \%)$ & \\
\hline Status of Diabetes & & & & & 0 \\
\hline Controlled & $17(34 \%)$ & $13(26 \%)$ & $2(4 \%)$ & $0(0 \%)$ & \\
\hline Uncontrolled & $0(0 \%)$ & $2(4 \%)$ & $12(24 \%)$ & $4(8 \%)$ & \\
\hline
\end{tabular}

Chi-Square test was applied, P-Value $<0.05 \%$ was taken as significant. 


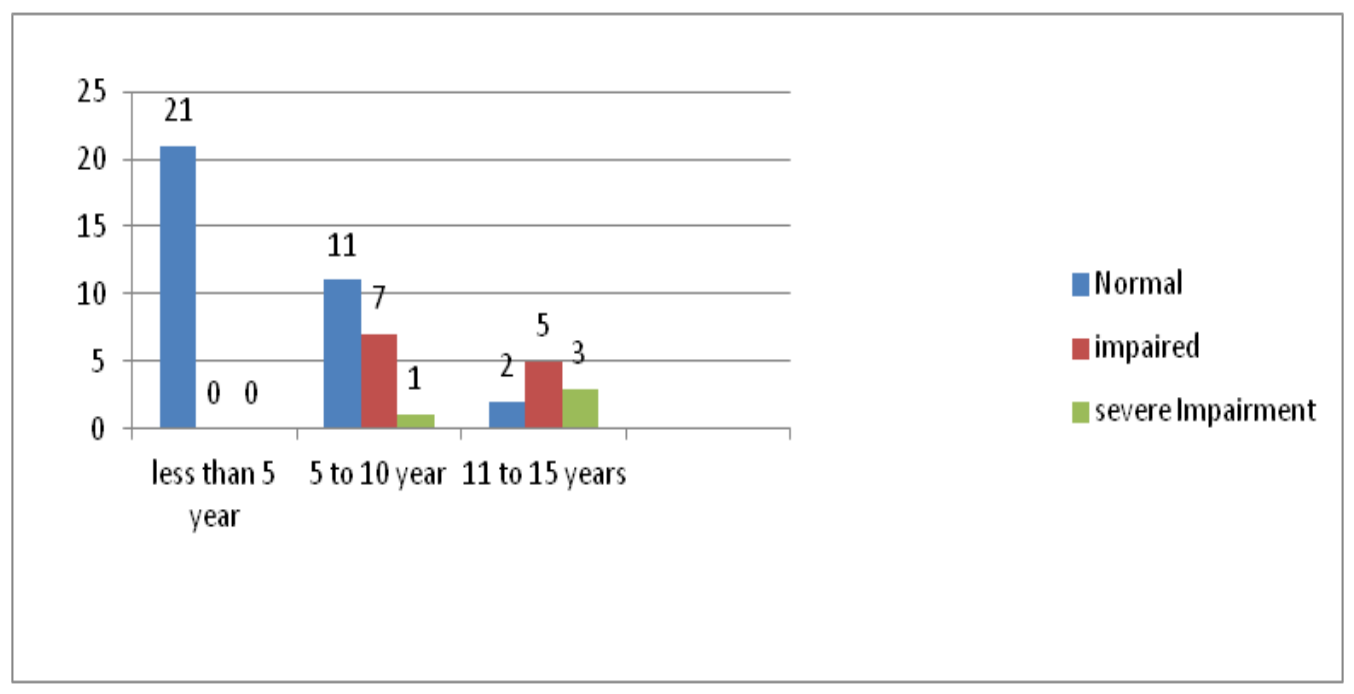

Figure I Color vision, duration of Diabetes.

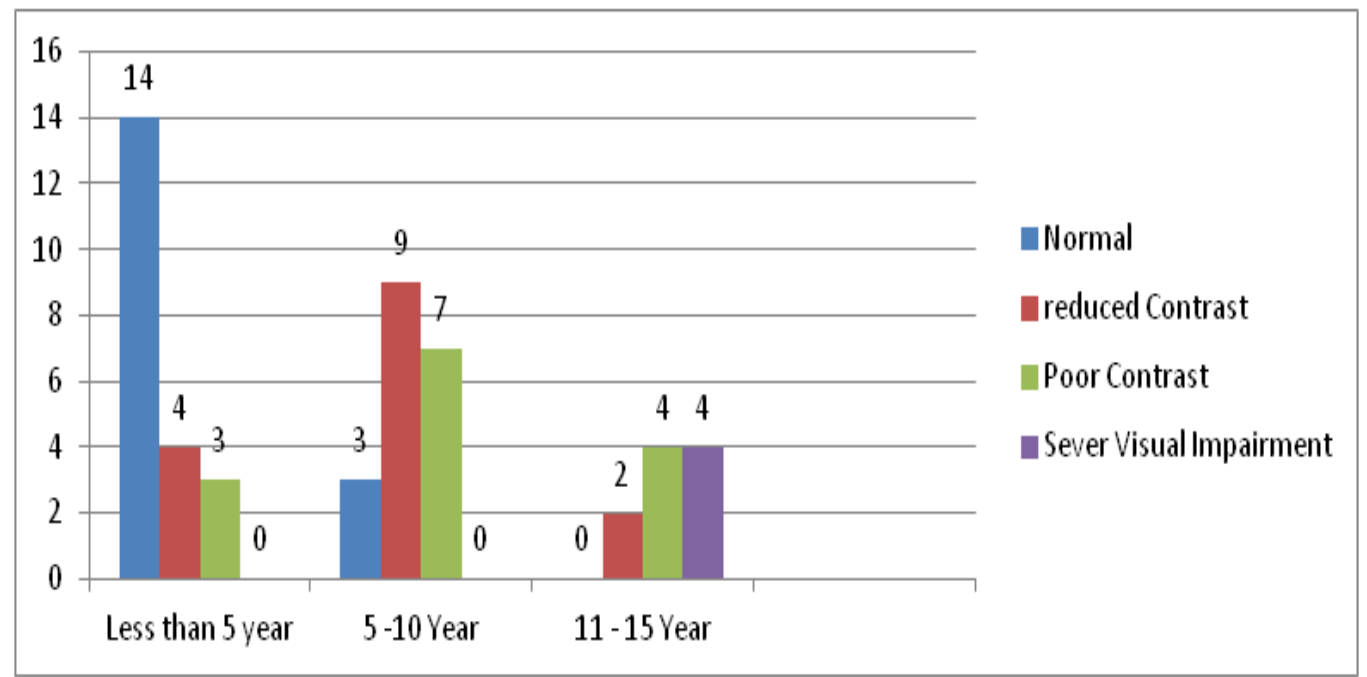

Figure 2 Contrast sensitivity, duration of diabetes.

Dr. Rashmi, et al. also conducted a similar study to evaluate the status of contrast sensitivity and its correlation with the duration of diabetes and glycemic control. The result of their study showed the similar results with my study that contrast sensitivity was significantly lower (Pvalue 0.03) in diabetic patients without retinopathy. ${ }^{6}$ Virotti A, et al. ${ }^{7}$ also conducted a similar study to measure the contrast sensitivity in diabetic patients with and without retinopathy and also analyze the relationship with the glycemic control. The results showed that contrast sensitivity was decreases in diabetic patients without diabetic retinopathy when compared with control groups. The result of their study showed a definite relation between contrast sensitivity with the status of diabetes. ${ }^{7}$ Another study showed the similar results with my study. That study evaluates the color vision contrast sensitivity and visual acuity in diabetic patients. The result showed that there was statistically significant loss in contrast sensitivity in diabetic eyes without retinopathy. ${ }^{3}$

The finding of my study showed that the color vision had a highly significant association ( $\mathrm{P}$ - value 0.00$)$ with the status of diabetes (controlled/uncontrolled). It has been reported in another study that the acquired color vision deficiency was significantly associated with the status of diabetes (Controlled/Uncontrolled). Acquired color vision deficiency was more in patients who had an uncontrolled high blood glucose level. It is considered that the status of diabetes is responsible for color vision. Macula is responsible for central vision which has a large number of the cones which help in color perception. Macula is more affected in uncontrolled diabetic patients that why the color vision deficiency is significantly higher in uncontrolled diabetic patients. Moreover, this study also showed that color vision had a highly significant association (P-value 0.00 ) with the duration of diabetes. It has been reported in another study the duration of diabetes showed a significant relationship with color vision. Higher the duration of diabetes may affect the color vision of the individual more. The duration of diabetes and uncontrolled diabetes mellitus may be significantly associated the color vision defect. When compare the findings with other study which showed that the acquired color vision deficiency was significantly associated with macular odema and diabetic maculopathy (Table 3).,34 
Table 3 Status of color vision with general variables of the patients $(n=50)$

\begin{tabular}{|c|c|c|c|c|}
\hline & \multicolumn{4}{|c|}{ ColorVision } \\
\hline & Normal & Impaired & Sever Impairment & P-Value \\
\hline Variables & $n(\%)$ & $\mathrm{n}(\%)$ & $n(\%)$ & \\
\hline Age & & & & 0.51 \\
\hline \multicolumn{5}{|c|}{ Mean age $48.42+5.15$} \\
\hline \multicolumn{5}{|l|}{ Gender } \\
\hline Male & $17(34 \%)$ & $4(8 \%)$ & $\mathrm{I}(2 \%)$ & 0.441 \\
\hline Female & 17 (34\%) & $8(16 \%)$ & $3(6 \%)$ & \\
\hline \multicolumn{5}{|l|}{ Duration } \\
\hline Less than 5 years & $21(42 \%)$ & $0(0 \%)$ & $0(0 \%)$ & \\
\hline 5- 10 years & II (22\%) & $7(14 \%)$ & $\mathrm{I}(2 \%)$ & 0 \\
\hline II- 15 years & $2(4 \%)$ & $5(10 \%)$ & $3(6 \%)$ & \\
\hline \multicolumn{5}{|l|}{ Type of Diabetes } \\
\hline Type I & $9(18 \%)$ & $10(20 \%)$ & $4(8 \%)$ & \\
\hline Type 2 & $22(44 \%)$ & $2(4 \%)$ & $0(0 \%)$ & 0.002 \\
\hline Dietary Control & $3(6 \%)$ & $0(0 \%)$ & $0(0 \%)$ & \\
\hline \multicolumn{5}{|l|}{ Status of Diabetes } \\
\hline Controlled & $32(64 \%)$ & $0(0 \%)$ & $0(0 \%)$ & 0 \\
\hline Uncontrolled & $2(4 \%)$ & $12(24 \%)$ & $4(8 \%)$ & \\
\hline
\end{tabular}

Chi- square test was applied. P-value $<0.05$ was taken as significant.

My study was found insignificant association of color vision and contrast sensitivity with age, gender and types of diabetes. The finding of our study were comparable with the finding of previous study showed that the diabetic status was not depend on age, gender , history of eye surgery and type of diabetes. ${ }^{3,6}$ Contrast sensitivity is the function of the retina. Therefore, contrast sensitivity measurement is the useful screening tool for the early detection of the retinopathy. It may also be helpful in detecting the relationship between metabolic control and retinal function. ${ }^{9}$ Another study suggested that reduced contrast sensitivity in diabetic patients were usually associated with high blood glucose level. ${ }^{10}$ My study included both type 1 and type 2 diabetes. Type 2 diabetes was found higher than type 1 diabetes. My study showed no statistically association of type of diabetes with contrast sensitivity impairment but an association of color vision with type of diabetes. In another study it was found that a significant association of color vision with type of diabetes. Color vision impairment was found in type 2 diabetes. ${ }^{11}$ But in contrast to other study it was found that there was no association of type of diabetes with color vision but an association with type of diabetes was found with contrast sensitivity. ${ }^{4}$ Therefore, a large sample size was required for confirmation the same. This study results emphasize that the diabetic mellitus can have an early neural abnormalities even in patients who have good visual acuity. Thus, color vision and contrast sensitivity measurements can provide very useful additional information about visual dysfunction in diabetes mellitus patients that cannot be obtained from visual acuity measurements alone.

\section{Conclusion}

There were highly significant association of Status of Diabetes (Controlled/uncontrolled) and duration of diabetes with color vision and contrast sensitivity. As the duration of diabetes increases color vision and contrast sensitivity would be impaired. There was no significant association of age and gender with color vision and contrast sensitivity. This study was helpful in detecting the early visual function compromised due to diabetes before the occurrence of diabetic retinopathy. ${ }^{12-15}$

\section{Acknowledgments}

None.

\section{Conflicts of interest}

There is no conflict of interest.

\section{References}

1. Beroz J, Royal C, Heading V. Ethnic identity and type 2diabetes. Journal of diabetic complication and Medicine. 2012;16:624-632.

2. Keratape AS, Kose S, Egrilmze S. Factor affecting contrast sensitivity in healthy individuals: A pilot study. Turk J Ophthalmol. 2017;47(2):80-84.

3. Heravian J, Shoeibi N, Azimi N, et al. Evaluation of contrast sensitivity, color vision and visual acuity in patients with and without retinopathy. Iranian Journal of Ophthalmology. 2010;22(3):33-40. 
4. Malik MY, Tariq H, Yasmeen A, et al. Impaired Color Vision and Contrast Sensitivity in Patients with Diabetes Mellitus. Pakistan Journal of Ophthalmology. 2018;34(1):30-37.

5. Vaibhavee N, Manisha S. A study of Contrast Sensitivity Changes in normal individual and Diabetic Patients with and without diabetic retinopathy. International Journal of Research and Medicine Sciences. 2017;5(11):4840-4845.

6. Rashmi S, Rejitha CV, Anupama B, et al. Contrast Sensitivity in Diabetic Patients without Retinopathy And Its Correlation with the Duration of Diabetes And Glycemic Control. IOSR Journal of Dental and Medical Sciences. 2016;15(8):11-13.

7. Virotti A, LobefaloL, Petitti MT. Relationship between Contras Sensitivity and metabolic control in diabetic with and without diabetic retinopathy. Ann Med. 1998;30(4):369-374.

8. Anton A, Bohringer D, Bach $\mathrm{M}$, et al. Contrast sensitivity with bifocal intraocular lenses is halved, as measured with the Freiburg Vision test, yet patients are happy. Grafes Arch Clin Exp Ophthalmol. 2014;252(3):539-544.
9. Khan MM, Mahmud S, Karim MS, et al. Case control study of suicide in Karachi, Pakistan. Br J Psychiatry. 2008;193(5):402-405.

10. Feitosa-Santana C, Oiwa NN, Paramei GV, et al. Color space distortions in patients with type 2 diabetes mellitus. Visual Neuroscience. 2006;23:663-668.

11. Moudgil T, Arora R, Kaur K. Prevalence of Color blindness in Children. International Journal of Medical and Dental Sciences. 2016;5(2).

12. Sara S, Anoushiravan R, Afsaneh R, et al. Contrast sensitivity in moderate and dim light conditions in patients with diabetes in the absence of diabetic retinopathy. BMJ Open Diab Rec Care. 2017;5.

13. Li-Ting T, Kuo-Meng L, Yuh J, et al. Detecting Visual function Abnormality with the contrast Dependent Visual test in patients with type 2 Diabetes. PLoS One. 2016;11(9):e0162383.

14. Gauri SS, Raju K. Visual functions and disability in diabetic retinopathy patients. Jouranl of Ophthalmology. 2014;7(1):37-43.

15. Dean L, McEntyre J. The genetic landscape of diabetes. National Information of Biotechnology Information. 2004. 